\title{
A acessibilidade nos serviços de saúde sob a perspectiva da pessoa com deficiência, Recife - PE
}

\author{
Accessibility in health services from the perspective of people with disabilities, Recife - PE \\ Accesibilidad en los servicios de salud desde la perspectiva de las personas con \\ discapacidad, Recife - PE
}

\begin{abstract}
Maria Eduarda Lima de Carvalho ${ }^{1 *}$, Tereza Natália Bezerra de Lima ${ }^{1}$, Joelma Laurentino Martins de Souza1, Tânia Maria Lago Falcão ${ }^{1}$ Ilka Jenifer Menezes Taurino², Andréia Patrícia Terenci², Ianne Larisse Alves Ferreira ${ }^{3}$, Débora Rafaela Amorim Ferreira ${ }^{4}$, Deiviane Lucio Fernandes ${ }^{4}$, Mayra Salgado de Lucena ${ }^{5}$, Julia de Lima Cabral ${ }^{6}$, Fernanda Barbosa dos Santos ${ }^{7}$.
\end{abstract}

\section{RESUMO}

Objetivo: Analisar as condições de acessibilidade aos serviços de saúde, vivenciadas por pessoas com deficiência, predominantemente nas unidades básicas de saúde no estado de Pernambuco. Métodos: Tratase de uma pesquisa de natureza exploratória e de abordagem qualitativa. A população estudada foi formada por pessoas com algum tipo de deficiência sensorial (visual/auditiva) ou física, residentes de área coberta, usuárias ou não dos serviços de saúde. As entrevistas foram realizadas sob a anuência do entrevistado e, posteriormente, transcritos para análise. Foram entrevistados doze usuários com deficiência: o primeiro participante foi escolhido por um profissional de uma das unidades de saúde, e os demais foram definidos a partir da técnica de amostragem snowball. Resultados: A análise dos dados foi feita através da análise de discurso, cujas ideias centrais originaram duas grandes categorias: Acessibilidade aos serviços de saúde e a Rede de Cuidados a Pessoa com Deficiência. Conclusão: As limitações de acessibilidade aos serviços de saúde foram relatadas pelos entrevistados, englobando barreiras tanto físicas quanto atitudinais. Foi identificada a relevância da escuta das pessoas com deficiência para a discussão e elaboração de ações para a prática do cuidado integral à saúde de tal parcela da população.

Descritores: Acesso aos serviços de saúde, Pessoas com deficiência, Saúde da pessoa com deficiência, Pesquisa qualitativa.

\begin{abstract}
Objective: To analyze the conditions of accessibility to health services experienced by people with disabilities, predominantly in basic health units in the state of Pernambuco. Methods: This is an exploratory research with a qualitative approach. The study population consisted of people with some type of sensory (visual / auditory) or physical disability, residents of covered areas, users or not of health services. The interviews were conducted under the consent of the interviewee and later transcribed for analysis. Twelve users with disabilities were interviewed: the first participant was chosen by a professional from one of the health units, and the others were defined using the snowball sampling technique. Results: Data analysis was performed through discourse analysis, whose central ideas originated two broad categories: Accessibility to health services and the Disability Care Network. Conclusion: The limitations of accessibility to health services were reported by
\end{abstract}

\footnotetext{
1 Universidade de Pernambuco - UPE, Recife-PE. *E-mail: melc.duda@gmail.com

${ }^{2}$ Centro de Formação Pós-graduação e Pesquisa em Saúde (CEFAPP), Recife-PE.

${ }^{3}$ Faculdade São Miguel, Recife-PE.

${ }^{4}$ Faculdade Metropolitana de Ciência e Tecnologia - FAMEC, Parnamirim-RN.

${ }^{5}$ Centro Universitário São Camilo, Crato-CE

${ }^{6}$ Centro Universitário do Vale do Ipojuca (UNIFAVIP/Wyden), Caruaru-PE.

7 Universidade Federal de Pernambuco - UFPE, Recife-PE.

${ }^{8}$ Fundação de Ensino Superior de Olinda - FUNESO, Olinda-PE.
} 
respondents, encompassing both physical and attitudinal barriers. It was identified the relevance of listening to people with disabilities for the discussion and elaboration of actions for the practice of integral health care of such part of the population.

Keywords: Access to health services, People with disabilities, Health of the disabled, Qualitative research.

\section{RESUMEN}

Objetivo: analizar las condiciones de accesibilidad a los servicios de salud que experimentan las personas con discapacidad, predominantemente en unidades básicas de salud en el estado de Pernambuco. Métodos: Esta es una investigación exploratoria con un enfoque cualitativo. La población de estudio consistió en personas con algún tipo de discapacidad sensorial (visual / auditiva) o física, residentes de áreas cubiertas, usuarios o no de servicios de salud. Las entrevistas se realizaron bajo el consentimiento del entrevistado y luego se transcribieron para su análisis. Se entrevistó a doce usuarios con discapacidades: el primer participante fue elegido por un profesional de una de las unidades de salud, y los otros se definieron utilizando la técnica de muestreo de bolas de nieve. Resultados: El análisis de datos se realizó a través del análisis del discurso, cuyas ideas centrales originaron dos amplias categorías: Accesibilidad a los servicios de salud y la Red de Atención de Discapacidades. Conclusión: Los encuestados informaron sobre las limitaciones de accesibilidad a los servicios de salud, que abarcan barreras físicas y de actitud. Se identificó la relevancia de escuchar a las personas con discapacidad para la discusión y elaboración de acciones para la práctica de la atención integral de salud de dicha parte de la población.

Palabras clave: Acceso a servicios de salud, Personas con discapacidad, Salud de los discapacitados, Investigación cualitativa.

\section{INTRODUÇÃO}

A pessoa com deficiência $(\mathrm{PcD})$ está historicamente inserida em um contexto social de luta e movimentos por inclusão na sociedade. Com um propósito claro de proteger a saúde da pessoa com deficiência, desde a prevenção até a reabilitação na sua capacidade funcional e desempenho humano, a Política Nacional de Saúde da Pessoa com Deficicência, foi instituída pela portaria do Ministério da Saúde, MS/GM no 1.060, de 5 de junho de 2002 (BRASIL, 2002). E dentre as principais diretrizes, a atenção integral à saúde e a promoção da qualidade de vida visam assegurar a igualdade de oportunidades às pessoas com deficiência, para a prática do direito à saúde (BRASIL, 2006).

A equidade, um dos pilares do SUS, diferente da igualdade, é entendida como igualdade de oportunidades, conferindo um princípio de justiça social. Caracterizada como "discriminação positiva", segundo Carneiro e Elias (2006), a equidade é direcionada a segmentos populacionais desfavorecidos socialmente, com políticas de atenção e planejamento que busquem eliminar tais desigualdades. Nesse contexto, para a pessoa com deficiência, ter as mesmas oportunidades quando não há como vivenciá-las, não é suficiente, comparativamente a alguém que não tenha tais limitações.

O perfil da assistência à saúde da pessoa com deficiência no Brasil, ainda é desarticulada, frágil e descontínua quanto às ações nas esferas pública e privada (GIRONDI JBR e SANTOS SMA, 2011). Nesse sentido, importa a implementação de políticas que garantam a ampliação no acesso aos serviços. As políticas assistenciais afirmam que a atenção básica é a porta de entrada para os demais serviços de saúde, no entanto, os usuários com deficiência parecem não encontrar esse acesso. Ainda nesse contexto, Othero MB e Dalmaso SW (2000) afirmam, com relação à saúde da pessoa com deficiência, que o quesito acesso foi identificado como principal necessidade dessa população.

O conceito de acesso é complexo a ponto de gerar imprecisão e pouca clareza quando empregado no âmbito dos serviços de saúde (Travassos C e Martins M, 2004). Para Donabedian A (2003), existem semelhanças entre os significados de acesso e acessibilidade, em se tratando de ações e serviços de saúde. Acesso se refere à possibilidade de alcance aos cuidados de saúde, de modo fácil e viável, relacionando-se à capacidade de oferta de serviços e de atendimento às necessidades de saúde de determinada população. 
Quando se trata de acessibilidade, considera-se também a disponibilidade de recursos em dado tempo e lugar; nesse caso, temos os aspectos dos serviços que exercem impacto e repercussão na possibilidade de uso pela população.

A acessibilidade conforma duas dimensões: a geográfica, quando se refere à distância espacial, estrutura e tempo de locomoção até o serviço; e a dimensão sócio organizacional, na qual é evidenciada a importância da adequação e/ou qualificação dos profissionais de saúde e de recursos tecnológicos utilizados para otimizar a interação profissional-usuário (DONABEDIAN A, 2003). Portanto, é importante destacar que a discussão acerca da temática de acesso da pessoa com deficiência, deve focar na realidade das barreiras arquitetônicas, ambientais, sem descuidar dos aspectos relacionados aos atores envolvidos nas barreiras atitudinais. A atenção primária, para Othero MB e Dalmaso SW (2009), representa o espaço adequado para ações que não estão relacionadas somente a aspectos biológicos das patologias, permitindo uma ampliação da cobertura assistencial. Oliver FC et al (1999), baseando-se nas referências da Reabilitação Baseada na Comunidade (RBC), aponta que um dos principais objetivos na atenção primária, com relação à saúde das pessoas com deficiência, é promover visibilidade social, construindo a inclusão a partir da valorização de processos e recursos locais.

Nesse sentido, este estudo objetiva analisar as condições de acessibilidade aos serviços de saúde, vivenciadas por pessoas com deficiência, predominantemente nas unidades básicas de saúde no estado de Pernambuco. Parte-se do pressuposto de que a atenção à saúde da população de pessoas com deficiência deve ser efetuada primordialmente na atenção primária, como apontam diversos autores.

\section{MÉTODOS}

Trata-se de uma pesquisa de natureza exploratória, com abordagem qualitativa e cuja análise dos dados coletados foi realizada através da análise de discurso. Utilizou-se a técnica de amostragem Snowball, na qual, segundo Victora CG et al (2000), cada entrevistado indica novas pessoas e, dessa forma, a amostra tende a crescer, até chegar ao ponto de saturação. Como critérios de inclusão, considerou-se: ser pessoa com deficiência, com idade igual ou maior de 18 anos, com autonomia pessoal e social, que receba ou não assistência em unidades de saúde. Como critérios de exclusão, considerou-se: pessoas com deficiência intelectual, justificada pela restrição na autonomia pessoal para responder a entrevista, e pessoas que se recusassem.

Todos os participantes assinaram o Termo de Consentimento Livre e Esclarecido (TCLE) e, pessoas com deficiência auditiva foram auxiliadas por intérprete de LIBRAS, que também se submeteu ao mesmo TCLE. Como instrumentos de coleta, foram utilizadas a entrevista semiestruturada, guiada por roteiro elaborado pela própria pesquisadora, e a observação direta. Foram realizadas doze entrevistas, sendo abordadas diferentes deficiências.

O roteiro continha questões relacionadas à acessibilidade, ao atendimento realizado pelos profissionais das Equipes de Saúde da Família (ESF) e pelo Núcleo de Apoio de Saúde da Família (NASF), e o sistema de saúde diante do usuário com deficiência. As informações foram registradas em diário de campo, pela observação direta dos informantes, além de gravadas.

Todo o material analisado foi alocado em categorias temáticas. As ideias centrais das respostas obtidas através dos relatos foram organizadas em duas dimensões, sendo identificadas duas grandes categorias: Acessibilidade aos serviços de saúde (relacionando-se a subcategorias: barreiras físicas de acesso e deslocamento para o serviço, barreiras atitudinais, necessidade de acompanhante e sentimentos dos usuários com deficiência) e a Rede de Cuidados a Pessoa com Deficiência, (relacionando-se a assistência oferecida pelos serviços e a sua utilização).

O estudo foi aprovado pelo Comitê de Ética em Pesquisa da Universidade de Pernambuco - UPE, com o número do parecer: 2.415.799, e a aplicação dos procedimentos de investigação, foram respeitados os valores éticos, através da Resolução 196/96 do Conselho Nacional de Saúde. 
Tabela 1 - Características sociodemográficas dos informantes.

\begin{tabular}{|c|c|c|c|c|c|c|c|c|c|}
\hline & Idade & Sexo & Escolaridade & Ocupação & $\begin{array}{l}\text { Estado } \\
\text { civil }\end{array}$ & Religião & $\begin{array}{c}\text { Tipo de } \\
\text { deficiência }\end{array}$ & $\begin{array}{c}\text { Área Coberta } \\
\text { por ESF e eNasf }\end{array}$ & $\begin{array}{c}\text { Acompanhado } \\
\text { pela ESF }\end{array}$ \\
\hline $\mathrm{E} 1$ & 35 & M & Fundamental Incompleto & Não & Casado & Evangélico & Física & $\operatorname{Sim}$ & Parcialmente* \\
\hline E2 & 48 & M & Superior Incompleto & Não & Solteiro & Não praticante & Física & Sim & Sim \\
\hline E3 & 35 & $\mathrm{~F}$ & Fundamental Incompleto & Não & Solteira & Evangélica & Auditiva & Sim & Sim \\
\hline E4 & 47 & M & Superior Incompleto & Sim & Casado & Evangélico & Visual & Sim & Não \\
\hline E5 & 42 & M & Médio Completo & Não & Casado & Evangélico & Física & Sim & Parcialmente $^{*}$ \\
\hline E6 & 36 & $\mathrm{~F}$ & Médio Completo & Sim & Casada & Católica & Visual & Sim & Parcialmente* \\
\hline E7 & 64 & M & Médio Incompleto & Aposentado & Casado & Católico & Visual & $\operatorname{Sim}$ & Parcialmente* \\
\hline E8 & 21 & $\mathrm{~F}$ & Médio Incompleto & Não & Casada & Evangélica & Auditiva & Sim & Sim \\
\hline E9 & 30 & M & Fundamental Incompleto & Não & Solteiro & Evangélico & Auditivo & Sim & Parcialmente* \\
\hline E10 & 25 & $\mathrm{~F}$ & Médio Completo & Não & Casada & Evangélica & Auditiva & Sim & Não \\
\hline E11 & 36 & M & Médio Incompleto & Sim & Divorciado & Evangélico & Auditivo & Sim & Parcialmente* \\
\hline E12 & 34 & $\mathrm{~F}$ & Médio completo & Sim & Casada & Evangélica & Física & Sim & Parcialmente* \\
\hline
\end{tabular}

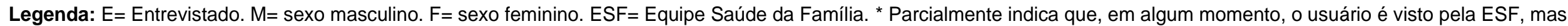
de maneira não sistemática.

Fonte: Carvalho MEL, et al., 2019. 


\section{RESULTADOS E DISCUSSÕES}

Foram entrevistados doze usuários com deficiência, residentes no Distrito Sanitário IV do município de Recife. Com a finalidade de condensar algumas informações acerca dos aspectos socio demográficos dos entrevistados, foi criado o quadro I, com o propósito de personificar os atores da pesquisa (Tabela 1).

Conforme mostra a Tabela 1, dentre outras variáveis, podemos destacar a escolaridade, na qual a maioria concluiu o ensino médio e poucos têm ocupação, os que trabalham são envolvidos com a temática. Todos com deficiência auditiva são evangélicos na Igreja Evangélica Batista, que dispõe de intérprete de LIBRAS em suas unidades, o que favorece acolhimento e inserção social dos surdos. Quanto à cobertura palas ESFs, foi identificada uma disparidade, todos são residentes de área coberta pelo programa, mas somente alguns consideram-se acompanhados pelos profissionais das equipes de atenção básica.

Como referido anteriormente, duas grandes categorias emergiram da análise, de forma coerente com os objetivos propostos para o presente estudo.

\section{Categoria I - Acessibilidade aos serviços.}

Esta categoria comporta quatro subcategorias, que se retroalimentam: as barreiras arquitetônicas no deslocamento para os estabelecimentos de saúde e em seu interior, o comprometimento da autonomia para o cuidado da saúde, as barreiras atitudinais dos profissionais e os sentimentos que esses usuários expressam frente à assistência recebida.

No que se refere ao deslocamento até os serviços de saúde, os entrevistados com deficiência física e visual frisaram as barreiras arquitetônicas encontradas, dificultando a sua chegada até a unidade. Como coloca Pagliuca LMF (2006), as inúmeras dificuldades nesse quesito, são reconhecidas nos dados do próprio Ministério da Saúde, segundo os quais de $10 \%$ de pessoas com deficiência, apenas $2 \%$ recebem assistência à saúde, devido a esse problema estrutural.

[E.6] "...infelizmente aqui, a acessibilidade nas ruas, cada dia ta mais precária, muitos buracos nas calçadas e ruas..."

[E.12] “E o transporte é aquele 'Deus nos acuda', né? Tem ônibus que não sei como ainda roda, caindo pedaços! E as estação, tudo sem nenhuma acessibilidade!"

O Decreto 5.296 de 02 de dezembro de 2004 delibera que a construção, reforma ou ampliação de projetos urbanisticos e edificações, deverão ser executadas de maneira acessível às pessoas com deficiência ou mobilidade reduzida (BRASIL, 2004). Além disso, em seu artigo 34, considera o transporte coletivo acessível, garantindo o uso pleno com segurança e autonomia por todos. Dessa forma, evidencia-se a fragilidade na garantia desses direitos à tal segmento da população, inviabilizando o deslocamento do usuário até o serviço de saúde ou o obrigando a utilizar meios de transporte privado.

[E.1] “..., mas a doutora tá de licença, tem que ir lá no posto da Madalena, aí fica bem mais difícil porque tem que pegar dois ônibus, aí tô esperando a daqui chegar mesmo."

[E.2] Como quando acontece muito de tá quebrado o elevador do ônibus. Gente eu estudei, estudei pesquisando em casa como conserta, quais principais problemas que dá e aprendi como resolver. Aí quando o motorista diz 'ah, ta quebrado, não da' eu vou falar o que tem que fazer, tentar explicar, mas ninguém dá ouvido!

[E.5] "...Isso que tô dizendo, nem toda hora a pessoa tá podendo pagar um carro pra levar, agora mesmo to querendo voltar a fisioterapia, tô muito parado..."

Além de encontradas nas ruas, calçadas e no uso de transporte coletivo, as barreiras físicas referentes ao acesso aos serviços de saúde também se estendem até ao próprio ambiente interno dos estabelecimentos. Condições como essas favorecem a sensação de falta de respeito e dignidade com os usuários com deficiência, enquanto cidadãos, de maneira que exprimem nos discursos: 
[E.4] "A própria sinalização na unidade, se é atendimento por meio de painel eletrônico, a gente cego não tem acesso, precisa ser sonorizado, que informe pra que a gente se dirija a sala tal, com autonomia."

[E.4] As portas, o que tem nelas não está em braile, a numeração não está em braile, não tem letras com ampliação pra quem é com baixa visão, os indicadores com cores diferenciadas com baixa visão, então assim, a sinalização, $90 \%$ das unidades não tem!

[E.12] "E mesmo com uma equipe ótima e tudo, se eu precisasse ir no banheiro tinha que descer tudinho e ir no térreo pra o único banheiro que a cadeira entrava, mas com porta aberta."

[E.12] Eles fizeram lá [Hospital da Mulher] o mamógrafo adaptado sim, pra mulher com deficiência né? Mas não fizeram uma maca não. Então se a gente precisar fazer um ultrassom, um exame intravaginal, porque a gente faz sexo! Então, não tem, eles não pensam além sabe?

Os relatos dos entrevistados corroboram com os achados de outro estudo, em que as condições de acessibilidade no ambiente interno das Unidades Básicas de Saúde não condizem com as normas técnicas preconizadas pela ABNT (ABNT, 2017). Segundo França ISX et al (2010), é perceptível uma preocupação dos gestores em relação ao acesso das pessoas com deficiência às unidades, no entanto, as soluções apresentadas, de fato, não atendem às suas necessidades, afinal estão aquém das normas técnicas.

Em muitos relatos, encontra-se uma estreita relação entre dependência da pessoa com deficiência e a insegurança gerada pela inacessibilidade nos espaços, o que torna impraticável a autonomia. É comum o apoio dos profissionais aos familiares através da escuta e acolhimento, no entanto, essa prática pode colaborar para a continuidade de uma situação de dependência do usuário com deficiência (OTHERO MB e DALMASO SW, 2009).

[E.1] "Venho, venho sempre porque depois que eu cai da cadeira fiquei mais com medo ainda de andar só, aí venho sempre com meu sobrinho."

[E.4] "Fico nessa dependência de alguém, pra que me ajude e eu chegue na sala do atendimento."

[E.8] "Aqui a comunicação é sempre muito difícil, não tem intérprete [de LIBRAS], então minha mãe sempre tem que vir comigo."

Outro aspecto a ser destacado refere-se às barreiras atitudinais dos profissionais de saúde. Considerando que o cuidado em saúde tem sua prática a partir do contexto sócio-histórico-cultural, os profissionais de saúde sendo parte desse meio, ainda atuam com segregação diante da deficiência (OTHERO MB e DAMALSO SW, 2009). Dessa forma, o estigma e representações sociais estereotipadas da deficiência colaboram para que a atenção à saúde nesse segmento da população seja ainda mais complexa e desafiadora.

[E.2] "Eles não sabem, não sabem como se portar diante de uma pessoa tetra, como eu.

[E.6] "... quando ele [profissional médico] começa, eu peço licença e digo 'olha, ela apenas está me acompanhando, a consulta é comigo, então a minha deficiência é visual apenas, se o senhor falar comigo, me perguntar, eu vou saber dizer o que tenho...' até porque a pessoa que tá comigo não vai saber o que eu tô sentindo, né?"

[E.10] "Por isso que eu não gosto! Ele [profissional médico] só fala com a minha mãe, mas eu que preciso saber de minhas coisas, da minha saúde!"

Nos discursos apresentados nesse sentido, é possivel identificar a insatisfação dos usuários diante do atendimento recebido pelos profissionais dos serviços de saúde. Desse modo, fica clara a existência de 
barreiras atitudinais no processo comunicacional, comprometendo a integralidade da atenção à saúde e a autonomia e corresponsabilização do usuário no autocuidado.

[E.9] Eu preciso ter acesso, quero ter acesso pra eu me cuidar, mas como não existe comunicação, não tem como. Queria muito que tivesse uma intérprete de LIBRAS nos hospitais...

[E.10] "Deveria ter intérprete e eu não precisava chamar a minha mãe, eu resolveria sozinha."

[E.10] "Ah, eu fico muito incomodada! Fico querendo saber, curiosa pra entender o que o médico ta falando sobre minhas coisas...Minha mãe faz: 'perai' (gestos) e continua falando com ele, só eles dois."

No estudo de França ISX e Pagliuca LMF (2008), é destacada a necessidade de as instituições formadoras de profissionais de saúde assumirem a responsabilidade de desenvolver competências para ser estabelecida uma conciliação entre o profissional em formação e a realidade social, capacitando este para compreender e atuar como agente de transformação das práticas sociais e de si mesmo. Além disso, tal argumento condiz com princípios contidos na legislação do Decreto no $3.298 / 1999$, que aborda sobre a inclusão das pessoas com deficiência no contexto da saúde, habilitação e reabilitação (BRASIL, 1999); e da Lei oㅡ 10.436/2002, que dispõe sobre a difusão da LIBRAS (Língua Brasileira dos Sinais) (BRASIL, 20002).

[E.03]: "Aqui eu tô muito feliz que estou com ela [intérprete de LIBRAS da entrevista], mas nunca tem e minha mãe precisa ajudar... não entendo nada dos outros não."

[E.06] "Quando a gente vai nas clínicas [serviço privado], aí eu escuto meu nome, a pessoa já sente um ânimo sabe? Já dá uma alegria na gente! É horrível, quando não tem [sonorização]...”

[E.12] "Às vezes, quando a gente é tratado em um local bem, mesmo que a acessibilidade não seja total, a gente se sente bem, né? Quando é tratado assim, nem como coitado nem como melhor pela deficiência..."

A percepção de ser cidadão pelo reconhecimento de sua identidade como pessoa, modifica a ideia do usuário sobre si mesmo. A alegria expressada por E.6 e não ser tratado como 'coitado', como dito por E.12, exterioriza uma sensação de valorização do sujeito, que se torna participante dos processos.

\section{Categoria II - Rede de Cuidados a Pessoa com Deficiência.}

O Plano Nacional dos Direitos da Pessoa com Deficiência (Plano Viver sem Limites), instituído através do Decreto 7.612 em novembro de 2011, promove o exercício pleno dos direitos da pessoa com deficiência, através da integração de políticas, programas e ações com eixos de atuação (BRASIL, 2011). Dessa forma, surge a Rede de Cuidados à Pessoa com Deficiência, visando articular os serviços, garantindo ações de promoção e prevenção, assim como identificação precoce de deficiências, tratamento e reabilitação (EDUFMA, 2017). Para o funcionamento da Rede foram estabelecidos objetivos específicos e diretrizes que, mediante os discursos analisados no presente estudo, encontram-se desrespeitados na prática do cuidado ao usuário com deficiência.

Em relação à visita domiciliar, a maioria dos entrevistados relata carência de suporte dos profissionais de saúde de sua unidade de referência:

[E.1]: "No começo, a minha agente de saúde ia lá, mas ela não podia tá indo direto lá porque tinha que tá no posto e também tinha as outras casas pra visitar, as pessoas da comunidade começaram a reclamar"

[E.7] "Ninguém vem, enfermeiro, nada!

[E.11] "Não, a gente vai só no hospital mesmo. Eu nunca recebi visita não, moro com minha mãe e nunca vimos." 
Dentre as diretrizes da Rede, algumas delas, claramente, não estão sendo consideradas: equidade; diversificação das estratégias do cuidado; atenção humanizada e centrada nas necessidades das pessoas; respeito aos direitos humanos; garantia de autonomia, independência e de liberdade às pessoas com deficiência para fazerem suas próprias escolhas. Isto aparece também em outras falas, especialmente quando há necessidade premente da visita domiciliar:

[E.1] “Eu tenho também uma escara. O curativo quem faz é minha esposa."

[E.2] "O que eu fico chateado é quando a gente se opera, quando me operei, voltei pra casa e avisei e o posto não mandou ninguém em casa! Pra ver minha situação, minha barriga ponteada."

São muitos os relatos nos quais é identificada a fragmentação do cuidado prestado aos usuários com deficiência participantes da pesquisa, como no discurso acima. A implantação de estratégias de acolhimento, análise de vulnerabilidade, acompanhamento e cuidado à saúde na atenção domiciliar são exemplos das ações estratégicas propostas pela Rede (EDUFMA, 2017). Essas ações, no entanto, não estão sendo consideradas, conforme as falas dos entrevistados:

[E.1]: "Se pudesse melhorar a frequência das doutora nas casa... se tivesse um pouco mais de médico pra outras coisas também, porque muita gente precisa de 'fono', eu mesmo acho que preciso de novo, as vezes falando assim não sai..."

[E.2]: "Nada. Nada de fisioterapia, nada mesmo, se tiver dor, tomo remédio, cuido assim só."

No que concerne à Atenção Básica, conta-se com a Atenção Odontológica e o Núcleo de Apoio à Saúde da Família (NASF). Dessa forma, ações que requerem competências específicas de algumas profissões e atividades multidisciplinares deveriam ser garantidas com a articulação verdadeira e permanente entre as equipes e os núcleos. Diante do exposto em relação à assistência à saúde das pessoas com deficiência, apesar de várias queixas sobre as barreiras, os entrevistados enfatizam como maiores problemas, aqueles relacionados aos serviços de saúde, indicando a precarização dos serviços da rede, como as dificuldades para fazer exames, demora para marcação de consultas, escassez de materiais, falta de medicamentos e escassez de profissionais nos serviços e para as visitas domiciliares.

[E.1]: "As dificuldade é que sempre tem coisa faltando, as vezes a dentista tá de férias, o aparelho que tá quebrado, as vezes a água."

[E.2]: "Assim doutora, ainda é a questão dos exames mesmo, que não demorasse tanto pra marcar, com até anos, já esperei até três anos pelo resultado de um!"

[E.3]: “Faltando remédio, falta 'material' de medir pressão, falta médico também...”

Estes discursos indicam nitidamente a naturalização, pelos usuários e profissionais, da lógica vigente no sistema de saúde atual. Consultas pontuais, olhar biologizante, medicalização, solicitação de exames e utilização de recursos tecnológicos na prática do cuidado em saúde parecem ser mais valorizados do que a assistência realizada com integralidade e longitudinalidade.

\section{CONSIDERAÇÕES FINAIS}

Constata-se que ainda há muito o que ser melhorado de fato, para que sejam assegurados às PcD os direitos na assistência à saúde, garantindo o que o SUS preconiza como preceitos constitucionais, integralidade, equidade e universalidade. Sendo assim, recomenda-se a qualificação dos profissionais, com implementações de ações de educação permanente, com o objetivo de conhecer as limitações, assim como as peculiaridades acerca das deficiências e desenvolver a prática de acolhimento e atendimento voltados a essa população, garantindo desde o respeito aos direitos dos usuários enquanto cidadãos. Espera-se que os resultados apresentados possam contribuir para aprimorar a lógica e a abordagem no cuidado e assistência à saúde da população com deficiência, visto que foram relatadas e discutidas questões reais vivenciadas pela 
mesma. Por fim, é necessária uma prática voltada à resolutividade das dificuldades encontradas no acesso aos serviços de saúde, partindo dos gestores e profissionais de saúde, que devem buscar qualificar o olhar, com empatia, diante desse segmento da população.

\section{REFERÊNCIAS}

1. ASSOCIAÇÃO BRASILEIRA DE NORMAS TÉCNICAS - ABNT. NBR 9050. Acessibilidade a edificações, espaço, mobiliário e equipamento urbanos. In: Coordenadoria Nacional Para Integração da Pessoa Portadora de deficiência. CORDE. [online] [acesso 2017 set 15].

2. BRASIL. Ministério da Saúde. Portaria oㅜ 1.060, de 05 de junho de 2002. Aprova, na forma do Anexo desta Portaria, a Política Nacional de Saúde da Pessoa Portadora de Deficiência.

3. BRASIL. Ministério da Saúde. Secretaria de Atenção à Saúde. Departamento de Ações Programáticas Estratégicas. Política Nacional de Saúde da Pessoa com Deficiência. Ministério da Saúde, Secretaria de Atenção à Saúde, Departamento de Ações Programáticas Estratégicas, Brasília: Editora do Ministério da Saúde, 2010.

4. BRASIL. Decreto no 3.298, 20 de dezembro de 1999. Regulamenta a Lei no 7.853, de 24 de outubro de 1989, dispõe sobre a Política Nacional para a Integração da Pessoa Portadora de Deficiência, consolida as normas de proteção, e dá outras providências. Diário Oficial da União. 21 Dez 1999.

5. BRASIL. Lei no . 10.436, de 24 de abril de 2002. Dispõe sobre a Língua Brasileira de Sinais - Libras e dá outras providências. Diário Oficial da União. 25 Abr 2002.

6. BRASIL, Ministério da Saúde. Secretaria de Atenção à Saúde. Departamento de Ações Programáticas Estratégicas. Redes Estratégicas do SUS e Biopolítica: cartografias da gestão de políticas públicas. Ministério da Saúde, Secretaria de Atenção à Saúde, Departamento de Ações Programáticas Estratégicas, Brasília: Ministério da Saúde, 2016.

7. BRASIL. Decreto no 7.612, de 17 de novembro de 2011. Institui o Plano Nacional dos Direitos da Pessoa com Deficiência - Plano Viver sem Limite. Diário Oficial da União. 18 Nov 2011.

8. BRASIL. Ministério da Saúde. Secretaria de Atenção à Saúde. Departamento de Ações Programáticas Estratégicas. Manual de legislação em saúde da pessoa com deficiência / Ministério da Saúde, Secretaria de Atenção à Saúde, Departamento de Ações Programáticas Estratégicas. - 2. ed. rev. atual.- Brasília: Editora do Ministério da Saúde, 2006.

9. BRASIL. Decreto ํㅜ 5.296, de 02 de dezembro de 2004. Regulamenta as Leis nㅇ 10.048 , de 8 de novembro de 2000, que dá prioridade de atendimento às pessoas que especifica, e 10.098, de 19 de dezembro de 2000, que estabelece normas gerais e critérios básicos para a promoção da acessibilidade das pessoas portadoras de deficiência ou com mobilidade reduzida, e dá outras providências. Diário Oficial da União. 03 Dez 2004.

10. CARNEIRO JRN, ELIAS PE. Controle público e equidade no acesso a hospitais sob gestão pública não estatal. Rev Saude Publica, 2006; 40(5): 914-920.

11. DONABEDIAN, A. An introduction to quality assurance in health care. New York: Oxford University, 2003.

12. FRANÇA ISX et al. Violência Simbólica no acesso das pessoas com deficiência às unidades básicas de saúde. Revista Brasileira de Enfermagem, Brasília, 2010 dez; 63 (6): 964-971.

13. FRANÇA ISX, PAGLIUCA LMF. Acessibilidade das pessoas com deficiência ao sus: fragmentos históricos e desafios atuais. Revista RENE, Fortaleza, 2008 abr/jun; 9(2): 129-137.

14. GIRONDI JBR, SANTOS SMA. Deficiência física em idosos e acessibilidade na atenção básica em saúde: revisão integrativa da literatura. Revista Gaúcha de Enfermagem, 2011; 32(2):385.

15. MINAYO MCS. O desafio do conhecimento: Pesquisa qualitativa em saúde. 14 ed. São Paulo: Hucitec, 2014.

16. MINAYO MCS. Pesquisa social: teoria, método e criatividade. Petrópolis, RJ: Vozes Limitada, 2011.

17. OTHERO MB, DALMASO SW. Pessoas com deficiência na atenção primária: discurso e prática de profissionais em um centro de saúde-escola. Rev. Interface - Comunicação, Saúde, Educação, 2009 mar;13(28):177-188.

18. OLIVER FC et al. Reabilitação baseada na comunidade: discutindo estratégias de ação no contexto sociocultural. Rev. ter. ocup, 1999; 10(1): 1-10.

19. PAGLIUCA LMF. Mapeamento da acessibilidade do portador de limitação física a serviços básicos de saúde. Esc Anna Nery, 2006; 10(3): 494-500.

20. PAGLIUCA LMF et al. Acessibilidade e deficiência física: identificação de barreiras arquitetônicas em áreas internas de hospitais de Sobral, Ceará. Rev Esc Enferm USP, São Paulo, 2007; 41(4):581-8.

21. TRAVASSOS C, MARTINS M. Uma revisão sobre os conceitos de acesso e utilização de serviços de saúde. Cad. Saúde Pública, 2004; 20(2):190-198.

22. Universidade Federal do Maranhão, UNA-SUS/UFMA. Redes de Atenção à Saúde: Rede de Cuidado à Pessoa com Deficiência. São Luís: EDUFMA, 2017.

23. VÍCTORA CG et al. Pesquisa qualitativa em saúde: uma introdução ao tema. 1 ed. Porto Alegre: Tomo Editorial, 2000. 